\title{
La competencia informacional-digital en la enseñanza y aprendizaje de las ciencias en la educación secundaria obligatoria actual: una revisión teórica
}

\author{
Daniel Valverde-Crespo ${ }^{1, a}$, Antonio de Pro-Bueno ${ }^{1, b}$, Joaquín González-Sánchez ${ }^{2, c}$ \\ ${ }^{1}$ Departamento de Didáctica de las Ciencias Experimentales, Facultad de Educación. Universidad de Murcia. \\ Murcia. España. \\ ${ }^{2}$ Departamento de Quimica-Física, Facultad de Quimica. Universidad de Murcia. Murcia. España. \\ adaniel.valverde@um.es, ${ }^{\mathrm{b}}$ nono@um.es, ${ }^{\mathrm{c} j o s q u i n @ u m . e s ~}$
}

[Recibido: 14 marzo 2017. Revisado: 18 noviembre 2017. Aceptado: 25 enero 2018]

\begin{abstract}
Resumen: En este trabajo se realiza una revisión teórica sobre la competencia informacional-digital y su repercusión en la enseñanza de las Ciencias. Para ello, hemos tratado de aportar respuestas a las cuestiones: ¿es lo mismo competencia informacional que competencia digital?; ¿qué ha investigado la didáctica de las ciencias con respecto a la competencia informacional-digital?; ¿ ¿es esta competencia una parte de la competencia científica?; ¿tenemos ejemplos en el ámbito de la enseñanza y aprendizaje de las Ciencias? Nos hemos centrado en la Educación Secundaria Obligatoria, etapa en la que destaca el papel de esta competencia como elemento formativo de ciudadanos alfabetizados en búsqueda, selección y valoración objetiva y crítica de la información científica disponible en contextos digitales.
\end{abstract}

Palabras clave: Información digital; Competencias clave; TIC; Educación secundaria; Enseñanza de las ciencias.

The information and digital competence in Science Teaching in the current Secondary Education

Abstract: In this study, a theoretical review is made about the informational-digital competence and its repercussion in the teaching of Sciences. To do this, we have tried to provide answers to the questions: is the same information competence as digital competence? What has science education researched with respect to digital-informational competence? Is this competence a part of the scientific competence? Do we have examples in the field of teaching and learning of Sciences? We have focused on Compulsory Secondary Education, a stage in which it highlights the role of this competence as a training element for literate citizens in search, selection and objective and critical assessment of scientific information available in digital contexts.

Keywords: Online information; Key competences; ICT; Secondary education; Science teaching.

Para citar este artículo: Valverde-Crespo D., Pro-Bueno A., Gónzález-Sánchez, J. (2018) La competencia informacional-digital en la enseñanza y aprendizaje de las ciencias en la educación secundaria obligatoria actual: una revisión teórica. Revista Eureka sobre Enseñanza y Divulgación de las Ciencias 15(2), 2105. doi: 10.25267/Rev_Eureka_ensen_divulg_cienc.2018.v15.i2.2105

\section{Origen y justificación}

Las tecnologías de la información y la comunicación (TIC), las competencias básicas y los temas socio-científicos son tres de los paradigmas de la enseñanza de las ciencias señalados por Garritz (2010), que resultan esenciales para determinar las expectativas y objetivos de la educación científica en la actualidad así como en las próximas décadas.

Las TIC han cambiado nuestro estilo de vida, el trabajo y el ocio, las relaciones sociales o la forma de procesar la información por parte de los ciudadanos y, precisamente por la amplitud de sus repercusiones, pueden ser impulsoras potenciales de un cambio metodológico en las clases (Coll 2008) y obviamente en las de ciencias.

En cuanto a las competencias básicas y los temas socio-científicos, ambos deben formar parte de un proceso formativo que ha de contemplar, entre otros aspectos, la comprensión de la 
información, la capacidad de reflexionar críticamente sobre la misma, la comunicación a otros, la toma de decisiones o un posicionamiento argumentado frente a ella. El objetivo de todo lo anterior es formar una ciudadanía capaz, dialogante, solidaria, responsable y activa frente a problemas socio-científicos que afectan a la sociedad (Banet 2010).

En el ámbito de las ciencias, las últimas ediciones de la Encuesta de Percepción Social de la Ciencia de la Fundación Española para la Ciencia y la Tecnología (FECYT 2015, 2017) muestran que Internet se ha convertido en la primera fuente de información en dicho ámbito. Además, según estos estudios, los medios más habituales en contextos no especializados son: Wikipedia, los medios digitales generalistas, y más recientemente las redes sociales y Youtube. El estudio longitudinal realizado sobre los resultados de esta encuesta en sus sucesivas ediciones, llevado a cabo por Revuelta y Corchero (2015), también señala que las formas de buscar, acceder o recibir información científica han cambiado: asciende Internet y descienden las tradicionales (libros, revistas, enciclopedias) (Figura 1).

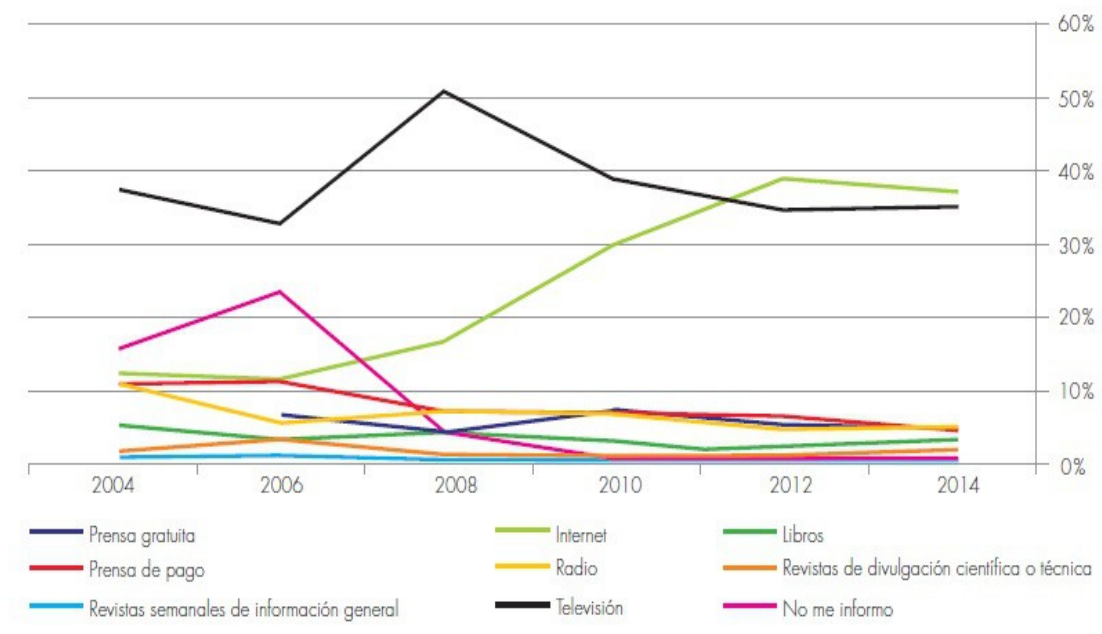

Internet

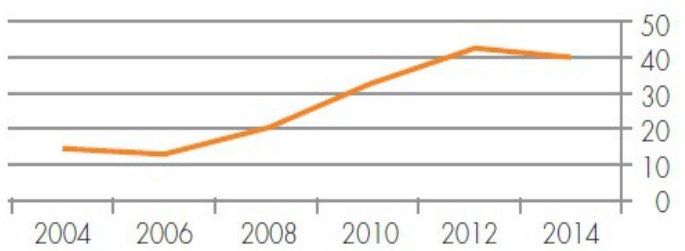

No me informo

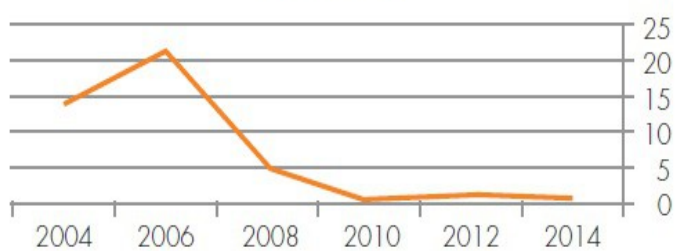

Figura 1. Gráficos extraídos de Revuelta y Corchero (2015) acerca del acceso a la información sobre ciencia.

El mismo estudio revela que esta tendencia es especialmente destacada en jóvenes de 15 a 24 años, frente a otros grupos de edad que permanecen más estancados (Figura 2).

Este cambio -la sustitución de unas fuentes y unos recursos por otros- resulta relevante en el proceso de aprender ciencias y debería ser tenido en cuenta, por lo menos en la educación obligatoria. Ahora bien, hablar sobre cómo utilizar Internet o la tecnología para acceder a información digital nos conduce a conceptos como la competencia digital o informacional (que discutiremos más adelante). Y, si consideramos la adquisición y desarrollo como elemento trasversal del currículum, deberíamos clarificar qué se puede y debe aportar desde cada una de las materias; en nuestro caso, desde las Ciencias Experimentales. 
Obviamente la inclusión de cualquier competencia debe tener en cuenta el contexto en el que situamos nuestra reflexión. Como indicaba Pedrinaci (2012), en este momento, existen problemas en torno a la ciencia escolar: se detecta una falta de interés del alumnado por la misma, le asignan una desproporcionada dificultad, hay una preocupante escasez en las vocaciones científicas... Y, entre las causas, se apunta a que lo que se enseña se encuentra alejado de las necesidades personales y sociales que tiene el alumnado en la actualidad.

Internet

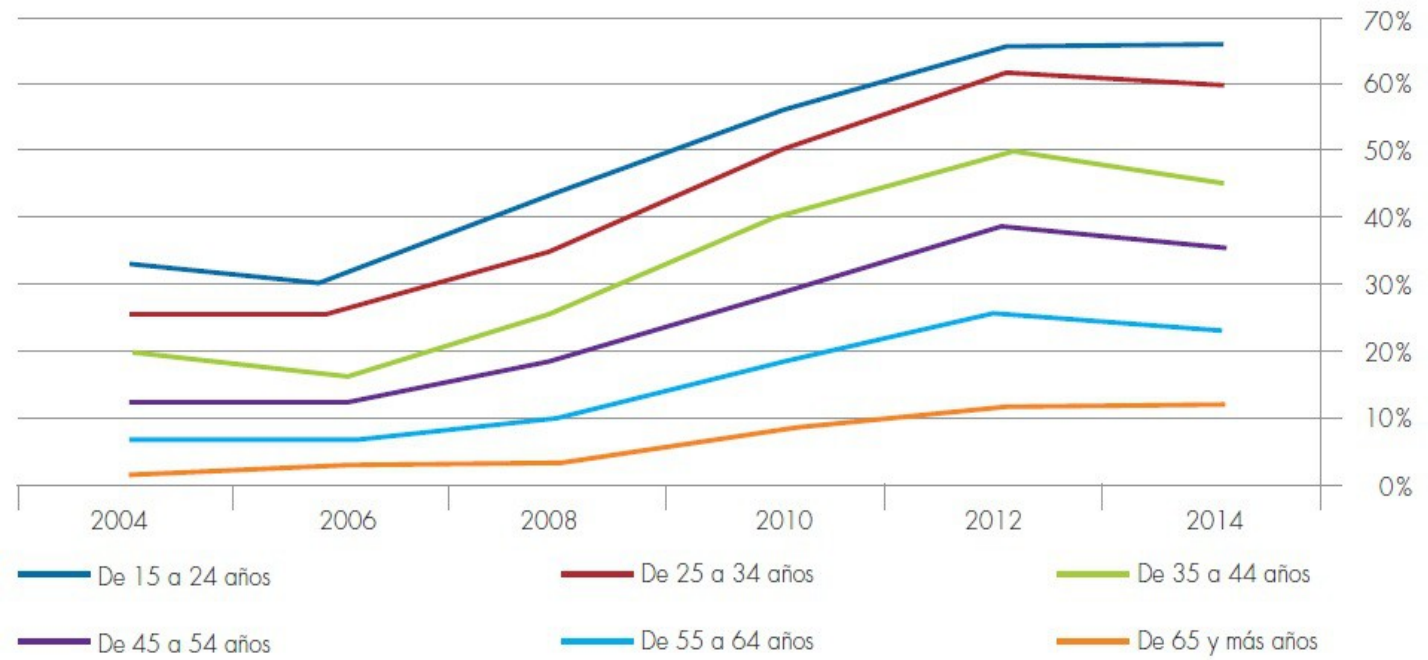

Figura 2. Gráfico extraído de Revuelta y Corchero (2015) sobre el uso de Internet según los rangos de edad.

Por otro lado, como señala De Pro (2012), se debe asumir que la ciencia que llega al estudiante no sólo proviene de los contenidos que se trabajan en las aulas, sino que también procede de fuentes como Internet, la televisión, el cine o la publicidad y la ciencia subyacente en estos medios no es fácil de relacionar con muchos de los conocimientos científicos que abordamos en enseñanza formal. Incluso, en nuestras propias clases de ciencias, a través de Internet, los estudiantes buscan y utilizan información de carácter científico. Por ello, es preciso formar al alumnado para hacer frente a estas situaciones y, para ello, la competencia informal-digital debe formar parte de la alfabetización científica de los ciudadanos (Annaniadou y Claro 2009).

Por ello, el objetivo de este trabajo es hacer una revisión teórica de los enfoques de dicha competencia en el ámbito de la Didáctica de las Ciencias Experimentales (DCE) y unas reflexiones sobre ellos. Hemos focalizado el estudio en la etapa de ESO porque la adquisición de las competencias básicas, entre las que se encuentra la competencia digital, es uno de los objetivos fundamentales de dicho nivel educativo.

\section{$¿$ Competencia informacional o competencia digital?... ¿Nos referimos a lo mismo?}

Las denominaciones competencia informacional y competencia digital forman parte de una terminología entrelazada y discutida (Campal 2015) y que engloba diversas habilidades digitales, lectoras, comunicativas, etc., muy distintas entre sí (Cid 2013). Por ello, resulta necesario en primer lugar aclarar qué se entiende por estos conceptos.

The European Parliament and the Council (2006) definía la competencia digital como «el uso seguro y crítico de las Tecnologías de la Sociedad y la Información, para el trabajo, el ocio y la comunicación» y se sustenta en el uso de las TIC para obtener, evaluar, crear, compartir información y comunicarse y colaborar a través de Internet. 
Posteriormente se desarrolla el marco conceptual DIGCOMP: A Framework for Developing and Understanding Digital Competence in Europe (Ferrari 2013) y su actualización DIGCOMP 2.0: The Digital Competence Framework for Citizens (Vuorikari, Punie, Carretero-Gómez y Van den Brande 2016) que supone un marco de consenso y de referencia a nivel europeo para la definición y comprensión de la competencia digital y para su desarrollo en los distintos currículos oficiales, programas... En este marco la competencia se divide en cinco áreas:

1. Información: identificar, localizar, recuperar, almacenar, organizar y analizar la información digital, evaluando su finalidad y relevancia.

2. Comunicación y colaboración: comunicarse en entornos digitales, compartir recursos a través de herramientas en línea, conectar y colaborar con otros a través de herramientas digitales, interactuar y participar en comunidades y redes, conciencia intercultural.

3. Creación de contenido digital: crear y editar nuevos contenidos (desde textos hasta imágenes y vídeos), integrar y reelaborar el conocimiento y el contenido previos, realizar producciones artísticas, contenidos multimedia y programación, saber aplicar los derechos de propiedad intelectual y las licencias de uso.

4. Seguridad: protección personal, protección de datos, protección de la identidad digital, medidas de seguridad, uso seguro y sostenible.

5. Resolución de problemas: identificar necesidades y recursos digitales, tomar decisiones informadas para elegir la herramienta más apropiada de acuerdo a la finalidad o necesidad, resolver problemas conceptuales a través de medios digitales, uso creativo de la tecnología, actualizar la competencia propia y la de otros.

Por ello, puede concluirse que en este marco de referencia, la competencia informacional forma parte de la competencia digital, que es un concepto mucho más amplio.

Cada una de las áreas presenta unas sub-competencias asociadas que permiten definirlas con mayor profundidad al establecer tres niveles (básico, intermedio y avanzado). Así las asociadas al área de información son:

1. Navegar, buscar y filtrar datos, información y contenidos digitales: Organizar y articular las necesidades de información, buscar datos, información y contenidos en entornos digitales, acceder a ellos y navegar entre ellos. Crear y actualizar estrategias personales de búsqueda.

2. Evaluación de datos, información y contenido digital: Analizar, comparar y evaluar críticamente la credibilidad y la fiabilidad de las fuentes de datos, información y contenido digital. Analizar, interpretar y evaluar críticamente los datos, la información y el contenido digital.

3. Gestión de datos, información y contenido digital: Organizar, almacenar y recuperar datos, información y contenido en entornos digitales. Organizarlos y procesarlos en un entorno estructurado.

El currículum oficial anterior (BOE 2007) introdujo por primera vez el Tratamiento de la información y competencia digital. Sin embargo, este "doble enfoque" fue criticado por Blasco y Durban (2012), ya que supone englobar dos competencias distintas, la informacional y la digital, con definiciones y articulaciones diferentes (aunque tengan elementos comunes). Además, esta asociación lleva aparejada una mayor dificultad en su comprensión y desarrollo práctico en las aulas. 
Posteriormente, en el actual currículum (BOE 2015), es renombrada como Competencia Digital y se define como aquella que «implica el uso creativo, crítico y seguro de las TIC para alcanzar los objetivos relacionados con el trabajo, la empleabilidad, el aprendizaje, el uso del tiempo libre, la inclusión y la participación en la sociedad». Además, se añade que «requiere de conocimientos relacionados con el lenguaje específico básico: textual, numérico, icónico, visual, gráfico y sonoro, así como sus pautas de decodificación y transferencia».

En ambos currículos, la competencia se aborda de forma transversal en todas las materias y abarca conocimientos y destrezas asociadas a la búsqueda y manejo de la información (de fuentes digitales o de otras) y otros del resto del ámbito digital: creación de contenidos en diferentes formatos digitales, resolución de problemas sencillos de hardware y software, uso seguro de la red, etc.

Otras aportaciones respecto a la competencia informacional inciden en su importancia en el aprendizaje autónomo y permanente de todo ciudadano para resolver problemas habituales en su contexto, y para lograr transformar la información en conocimiento útil (Area y Guarro, 2012; Monereo y Badia, 2012).

En conclusión, el área de información de la competencia digital -competencia informacionales un conjunto de conocimientos, procedimientos y actitudes necesarios para, de forma integrada, buscar, seleccionar, evaluar y gestionar información de fuentes digitales e Internet, y transformarla en conocimiento para tomar decisiones y resolver problemas en contextos variados y emergentes a nivel personal y social. Mientras que la competencia digital la engloba, pero incorpora otras que van más allá del ámbito informativo.

\section{¿Qué ha investigado la didáctica de las ciencias con respecto a la competencia informacional-digital?}

Las TIC e Internet están integradas en la vida de nuestro alumnado y son imprescindibles, dentro y fuera de las aulas. A pesar de ello, Badia (2009) y Monereo (2009) indican que los estudiantes tienen habilidades limitadas para buscar y seleccionar información en Internet y para transformarla en conocimiento; llevan a cabo un mero uso utilitarista de estos recursos para conseguir sus finalidades, sean educativas o no. Frente a estas consideraciones, hay otros que señalan que los estudiantes de secundaria muestran un buen manejo de la tecnología, sobre todo en sus hábitos cotidianos. Sin embargo, estos son insuficientes cuando aumenta la complejidad, como la evaluación de la información, la seguridad en la red o el uso ético de la tecnología (Calvani et al. 2012, Colás, Conde y Reyes 2017).

Con independencia de lo que saben, Monereo (2005) señala la necesidad de formar a nuestros estudiantes teniendo en cuenta ciertos riesgos existentes con el uso de Internet: los problemas de fiabilidad, el mercado en el que se ha convertido, la navegación desorientada, la presencia de información basura, el sesgo ideológico de la misma... Además, han surgido nuevas patologías asociadas a su utilización, como la «cibercondría», trastorno de ansiedad en la salud debido a la búsqueda de información en Internet de síntomas, enfermedades... (Starcevic y Aboujaoude, 2015). Parece necesario educar para hacer ciudadanos competentes en el manejo y consumo de información digital. Los autores también señalan que la forma más adecuada de aprender esta competencia es trabajarla, de forma intencionada y sistemática, en las aulas.

Sin embargo, el estudio de Grimalt, Pintó y Ametller (2013), sobre el uso de los recursos digitales en las aulas de ciencias en educación secundaria, muestra que es muy limitado y generalmente se reduce a fines expositivos, o para búsquedas cortas y concretas de información en Internet, sin aprovechar otras posibilidades didácticas (por ejemplo, la de indagar o reflexionar acerca de la información). Los autores sugieren que la integración de las 
TIC de forma efectiva debe acompañarse de un cambio en la forma de utilizarlas. Pero, dichos cambios, como indica Monereo (2010), son lentos y difíciles, debido a que los docentes encuentran obstáculos de carácter personal (inseguridad frente a cualquier cambio), profesional (desconocimiento de nuevas metodologías, recursos, formas de evaluación...) e institucional (falta de recursos necesarios, rechazo a las prácticas docentes innovadoras...).

Estas consideraciones se han visto corroboradas en investigaciones realizadas en el ámbito específico de la DCE. En la Tabla 1 se recogen trabajos de DCE en los que se ha investigado sobre la adquisición de competencia informacional por alumnos de secundaria.

Tabla 1. Aportaciones sobre habilidades de búsqueda, selección y evaluación de información en secundaria.

\begin{tabular}{|c|c|}
\hline Autor y año & Temática \\
\hline $\begin{array}{l}\text { Monereo y Fuentes } \\
(2008)\end{array}$ & $\begin{array}{l}\text { Investigación sobre los procesos y estrategias de alumnos de ESO para resolver sus } \\
\text { necesidades informativas, identificando las principales dificultades que tienen al hacer } \\
\text { frente a tareas diversas de búsqueda de información en Internet. }\end{array}$ \\
\hline $\begin{array}{l}\text { Walraven, Brand- } \\
\text { Gruwel y } \\
\text { Boshuizen (2009) }\end{array}$ & $\begin{array}{l}\text { La resolución de problemas de información y los tipos de criterios utilizan los estudiantes } \\
\text { de secundaria para evaluar los recursos y la información mientras buscan en Internet. }\end{array}$ \\
\hline $\begin{array}{l}\text { Julien y Barker } \\
(2009)\end{array}$ & $\begin{array}{l}\text { El análisis crítico de información científica relevante para su aprendizaje por parte de } \\
\text { estudiantes de secundaria y los criterios que utilizan para evaluar información científica } \\
\text { que encuentran en sus libros de texto y en otros medios populares incluyendo Internet. }\end{array}$ \\
\hline $\begin{array}{l}\text { Dimopoulos y } \\
\text { Asimakopoulos } \\
(2010)\end{array}$ & $\begin{array}{l}\text { Exploración de las pautas de navegación y de las características de las páginas web } \\
\text { preferidas por los estudiantes de secundaria cuando buscan información en la red sobre un } \\
\text { tema científico. }\end{array}$ \\
\hline $\begin{array}{l}\text { Álvarez-Herrero } \\
(2010)\end{array}$ & $\begin{array}{l}\text { La sobrecarga informativa a la que están expuestos los estudiantes de ESO y el desarrollo } \\
\text { de competencias digitales, a la vez que el aprendizaje de distintos conceptos de Física y } \\
\text { Química, que permitan llevar a cabo el proceso de construcción del conocimiento sin } \\
\text { interferencias por dicha sobrecarga informativa. }\end{array}$ \\
\hline $\begin{array}{l}\text { Tsai, Hsu y Tsai } \\
\text { (2012) }\end{array}$ & $\begin{array}{l}\text { La relación de las estrategias implícitas (auto-reflexión sobre la búsqueda, ensayo-error, } \\
\text { evaluación de las fuentes...) y las estrategias explícitas (palabras clave, cantidad de } \\
\text { resultados revisados, tiempo empleado...) mostradas por estudiantes de secundaria cuando } \\
\text { buscan información científica online. }\end{array}$ \\
\hline $\begin{array}{l}\text { Calvani, Fini, } \\
\text { Ranieri y Pici } \\
(2012)\end{array}$ & $\begin{array}{l}\text { La competencia digital del alumnado de secundaria en sus diferentes dimensiones } \\
\text { (conocimientos técnicos, comprensión de la tecnológia y sus implicaciones sociales, } \\
\text { habilidades cognitivas de alto orden...) }\end{array}$ \\
\hline $\begin{array}{l}\text { Colwell, Hunt y } \\
\text { Reinking (2013) }\end{array}$ & $\begin{array}{l}\text { Obstáculos encontrados al desarrollar habilidades, estrategias y disposiciones al hacer } \\
\text { investigaciones online (localizando información relevante, evaluando fuentes, sintetizando } \\
\text { información y comunicando resultados) por parte de estudiantes de secundaria. }\end{array}$ \\
\hline $\begin{array}{l}\text { Keck, Kammerer y } \\
\text { Starauschek (2015) }\end{array}$ & $\begin{array}{l}\text { La influencia de distintas fuentes de información y de sus expectativas de calidad al } \\
\text { localizar contradicciones en un texto científico online por parte de estudiantes de } \\
\text { secundaria. }\end{array}$ \\
\hline $\begin{array}{l}\text { Valverde-Crespo y } \\
\text { González-Sánchez } \\
\text { (2016) }\end{array}$ & $\begin{array}{l}\text { Percepciones de estudiantes de ESO y Bachillerato sobre Wikipedia como fuente de } \\
\text { información y la relación de éstas con lo expuesto en el currículo oficial en relación a la } \\
\text { competencia digital. }\end{array}$ \\
\hline $\begin{array}{l}\text { Valverde, González } \\
\text { y De Pro (2017) }\end{array}$ & $\begin{array}{l}\text { Competencias digitales del área de información manifestadas por estudiantes de ESO al } \\
\text { visualizar una animación sobre una reacción química a nivel miscroscópico. }\end{array}$ \\
\hline $\begin{array}{l}\text { Colás, Conde y } \\
\text { Reryes (2017) }\end{array}$ & Nivel de competencia digital percibido por el alumnado de niveles no universitarios. \\
\hline
\end{tabular}


Los trabajos centrados en la búsqueda de información -los de Monereo y Fuentes (2008) o Dimopoulos y Asimakopoulos (2010)- señalan que los estudiantes de secundaria son conscientes de algunos riesgos de Internet, como la fiabilidad de las fuentes. A pesar de ello, navegan de forma superficial y sin estrategias claras, de forma que, según los autores, asemejan su comportamiento al de ojear en un libro de texto buscando respuestas concretas y exactas para resolver sus tareas.

En este sentido, resulta relevante una conclusión de Colwell, Hunt y Reinking (2013): el uso de la tecnología que realizan los estudiantes fuera de las aulas les ha permitido desarrollar estrategias de bajo nivel para buscar, seleccionar y evaluar la información, pero éstas se encuentran muy interiorizadas y son muy difíciles de cambiar.

Por otro lado, la selección de las páginas web se basa especialmente en la posición en los buscadores de referencia, típicamente Google. Su uso está orientado a fines concretos (como la resolución de tareas) y no a la adquisición de conocimiento (Valverde-Crespo y GonzálezSánchez 2016). Esta situación se da en otros contextos (Julien y Barker 2009, Walraven et al. 2009, Tsai, Hsu y Tsai 2012), en ellos, el alumnado resulta poco reflexivo, valora únicamente los títulos de las webs, pone poco énfasis en la revisión del contenido, pero busca obtener la mayor calidad de la tarea que debe realizar, lo que resulta contradictorio.

Además, se han encontrado dificultades en los estudiantes para identificar información conflictiva o errónea en información digital -textual y visual- y también para interpretar su contenido (Keck et al. 2015, Valverde, González y De Pro 2017).

Por último, los resultados nos permiten afirmar, como Álvarez-Herrero (2010), que debemos enseñar a nuestros alumnos a realizar búsquedas en Internet que sean eficaces, de calidad y analíticas, para incentivar en ellos un juicio crítico que les permita diferenciar información fiable de la falsa, y a saber ordenar, estructurar y clasificarla. Sin embargo, hemos de ser precavidos por las dificultades que tiene esta acción didáctica.

\section{¿Son la competencia informacional y la competencia digital elementos específicos de la competencia científica?}

Quizás, el enfoque más extendido de la competencia científica es el que aporta el Programa de Evaluación Internacional de Estudiantes (PISA). En éste se formulan tres subcompetencias para su evaluación: Explicar fenómenos científicamente, Evaluar y diseñar experimentos y preguntas cientificas e Interpretar datos y pruebas cientificamente (OECD 2013) y cada una abarca unas habilidades. En la última subcompetencia, se contempla el uso de diversas fuentes (Internet, periódicos, televisión...) y sugiere que un individuo alfabetizado científicamente debe ser capaz de extraer la información, evaluar los argumentos y el contenido, en función de su base científica, y transferirlos a otras situaciones.

Pero, además de PISA, se han planteado otros enfoques en la DCE. Pedrinaci (2012) definía la competencia científica como un conjunto integrado de capacidades para utilizar el conocimiento con diferentes finalidades (describir, explicar y predecir fenómenos, formular hipótesis e investigar problemas científicos, documentarse...). Por ello, la formación debía conducir a que el alumnado fuera capaz de afrontar situaciones reales, resolver problemas y tomar decisiones relacionadas con la ciencia en los ámbitos personal, laboral y social, permitiendo el ejercicio de una ciudadanía responsable y satisfactoria.

En la misma línea, Cañal (2012) fijó las prioridades para personas científicamente competentes y, entre ellas, encontramos la capacidad de «buscar y seleccionar información relevante» relacionada con los procedimientos, y la de «valorar la calidad de una información en función 
de su procedencia y de los procedimientos utilizados para generarla» lo que se refiere a la naturaleza de la ciencia, como se recoge en la Tabla 2. Por lo tanto, pone de manifiesto que la competencia informacional y la competencia digital se incluyen en la competencia científica.

Tabla 2. Competencias informacionales y digitales integradas en la competencia científica (Cañal 2012).

\begin{tabular}{|c|c|c|}
\hline $\begin{array}{c}\text { Dimensión de la } \\
\text { competencia científica. }\end{array}$ & Capacidades científicas. & Aprendizajes básicos. \\
\hline Dimensión metodológica & \begin{tabular}{lcc} 
Capacidad & para & \multicolumn{2}{c}{ obtener } \\
información & relevante & para la \\
investigación & &
\end{tabular} & $\begin{array}{l}\text { Buscar y seleccionar fuentes de } \\
\text { información relevantes. } \\
\text { Obtener información fiable y relevante de } \\
\text { las distintas fuentes. }\end{array}$ \\
\hline Dimensión actitudinal & $\begin{array}{l}\text { Capacidad de valorar la calidad de } \\
\text { una información en función de su } \\
\text { procedencia y de } r \text { los } \\
\text { procedimientos utilizados para } \\
\text { generarla. }\end{array}$ & $\begin{array}{l}\text { Valorar positivamente las informaciones o } \\
\text { argumentos científicos frente a otros } \\
\text { contextos de investigación. } \\
\text { Valorar positivamente los procedimientos } \\
\text { científicos frente a otros que no lo sean, } \\
\text { en contextos de investigación. }\end{array}$ \\
\hline
\end{tabular}

Esta relación se considera clave desde muchos ámbitos, como muestra el estudio Delphi de Blanco-López, España-Ramos, González-García y Franco-Mariscal (2015). Su objetivo era determinar las competencias científicas clave para la ciudadanía propuestas por un grupo de expertos de diversos campos (científicos e ingenieros, investigadores en DCE, profesionales del sector privado, periodistas científicos...). Destacamos en sus resultados que, entre los cinco aspectos en los que existía consenso por el panel, se encontraba la competencia para buscar, analizar, sintetizar y comunicar información.

En consonancia con lo anterior, De Pro (2012) señala que la competencia informacional es una exigencia del conocimiento y la alfabetización científica puesto que, en las propias actividades de las clases de ciencias, es cada vez más necesaria la habilidad de búsqueda, selección y análisis de la información disponible. También destaca la importancia del uso de las TIC, mediante el planteamiento y realización en el aula de actividades y experiencias que las involucren de forma intencionada, progresiva y sistemática para fomentar su aprendizaje.

Por su parte, Franco-Mariscal (2015) propone un enfoque para el desarrollo de la competencia científica basada en la indagación; en la Tabla 3 recogemos las etapas propuestas por el autor. Como se ve, incluye el manejo de la información como elemento propio de la competencia científica.

Tabla 3. Dimensiones propuestas por Franco-Mariscal (2015) para el desarrollo de la competencia científica.

\section{Dimensiones de la competencia científica.}

1. Planteamiento de la investigación.

\section{Manejo de la información.}

Capacidad para buscar información en diferentes fuentes y valorarla de forma crítica y objetiva.

3. Planificación y diseño de la investigación.

4. Recogida y procesamiento de datos.

5. Análisis de datos y emisión de conclusiones.

6. Comunicación de los resultados de la investigación.

7. Actitud-reflexión crítica y trabajo en equipo. 
El autor considera que no se presta demasiada atención a la capacidad mencionada en las aulas de ciencias y, por ello, justifica su inclusión en base a dos argumentos: la considera deseable en la formación de personas capaces de afrontar de forma analítica y crítica la elevada cantidad de información científica disponible en la red en la actualidad; y considera su importancia para desarrollar capacidades científicas, ya que la búsqueda de la información, su análisis para valorar su fiabilidad o saber extraer contenidos relevantes para la investigación, son procedimientos básicos para construir marcos teóricos sólidos y coherentes, apoyados en fuentes contrastadas. El enfoque incluye las TIC en un entorno de aprendizaje por indagación, para desarrollar actitudes reflexivas y críticas hacia la información, como parte del proceso de investigación.

Por lo tanto, podemos decir que existe cierto consenso para afirmar que las competencias informacionales y digitales son elementos de la competencia científica.

\section{¿Hay ejemplos de propuestas y actividades para el desarrollo de la competencia científica que contemplen la competencia informacional-digital?}

A pesar del consenso sobre la necesidad de trabajar la competencia informacional-digital en las aulas de ciencias, en general no se le ha prestado la atención requerida, y la forma de trabajar con TIC no ha supuesto trasformaciones metodológicas relevantes (Grimalt, Pintó, Ametller 2013; Franco-Mariscal 2015). Ya hemos señalado que una causa sería la resistencia al cambio del profesorado (Gómez, Cañas, Gutiérrez, Martín-Díaz 2014; Gabby, Avargil, Herscovitz, Dori 2017).

No obstante, los docentes de ciencias comúnmente proponen búsquedas de información en Internet, pero sin formar previamente a los alumnos para ello y sin un fin claro de la actividad, por lo que como indican Gómez et al. (2014) conduce al aburrimiento del alumno, frustración del docente y fracaso de la actividad en el desarrollo de competencias.

Sin embargo, se pueden encontrar propuestas en las que se trabajan estas competencias. En la Tabla 4 se recogen algunas.

En la mayoría de las propuestas, se emplean contextos cotidianos o conocidos. Existe cierto consenso en que el empleo de este tipo de contextos (ahorro energético, educación ambiental, consumo de agua en botella, salud dental...), y la realización de preguntas investigables sobre ellos, permite el desarrollo de competencias digitales en el área de información (búsqueda y selección de fuentes, evaluación de la información, síntesis de la misma...) y conjuntamente permite el desarrollo de competencias científicas (argumentación en torno a un problema, toma de decisiones basadas en el conocimiento científico disponible, análisis de datos e información...). Los recursos digitales empleados son variados y diferentes según la propuesta (Internet como fuente de información, visionado de vídeos digitales, empleo de animaciones con contenido científico...).

Resulta destacable la aportación de Franco-Mariscal (2015) donde ejemplifica su enfoque alternativo para el desarrollo de la competencia científica mediante la exposición y evaluación de un trabajo de investigación sobre corrosión metálica llevado a cabo por alumnos de $3^{\circ}$ de ESO. Para desarrollar la dimensión manejo de la información se proporcionó a los alumnos criterios para facilitar la valoración de la fiabilidad de la información obtenida en la red (Fornás 2003). El autor señala que los alumnos fueron capaces de realizar marcos teóricos basados en fuentes fiables y con información pertinente y relevante, además de conocer por medio de la red trabajos de grupos de investigación sobre la temática.

Otra propuesta actual es el trabajo de Marzo y Monferrer (2015). Exponen y evalúan una propuesta basada en una metodología de indagación sobre formulación en química inorgánica 
para $3^{\circ}$ de ESO por medio de preguntas investigables relacionadas con ámbitos culturales o sociales de la temática. Se incluyen actividades que requieren la búsqueda y análisis de información en Internet para lo que emplea WebQuest como recurso para guiar al alumno en las búsquedas y que, a su vez, facilita la preparación de la propuesta por parte del docente.

Tabla 4. Resumen de propuestas de enseñanza que incluyen el desarrollo de la competencia informacional digital.

\begin{tabular}{|c|c|}
\hline Autor y año & Temática \\
\hline Murga-Menoyo y García (2010) & $\begin{array}{l}\text { Diseño, implementación y evaluación de una experiencia innovadora para la } \\
\text { Educación Ambiental formal. Uno de los objetivos persigue el desarrollo de } \\
\text { competencias para autonomía personal: capacidad de búsqueda de } \\
\text { información y autoorganización del trabajo en función de metas. }\end{array}$ \\
\hline López, Blanco y Haro (2011) & $\begin{array}{l}\text { Propuesta de una herramienta denominada «unidad didáctica web», con la } \\
\text { finalidad de desarrollar la competencia científica y el tratamiento de la } \\
\text { información y competencia digital en la ESO. Se toma como ejemplo el tema } \\
\text { del ahorro de energía. }\end{array}$ \\
\hline $\begin{array}{l}\text { Rodríguez, Blanco y Rueda } \\
\text { (2011) }\end{array}$ & $\begin{array}{l}\text { Propuesta de una unidad didáctica centrada en el consumo de agua } \\
\text { embotellada. Se presentan diversas actividades para el desarrollo de la } \\
\text { competencia científica, en las que se integradan las TIC, permitiendo } \\
\text { conjuntamente abordar el tratamiento de la información y competencia digital. }\end{array}$ \\
\hline $\begin{array}{l}\text { Franco, España y Blanco } \\
\text { (2014) }\end{array}$ & $\begin{array}{l}\text { Propuesta de una tarea que pretende que los estudiantes indaguen en la } \\
\text { influencia de algunas drogas en la aparición de caries, y que argumenten a favor } \\
\text { o en contra, aplicando los conocimientos científicos estudiados, y siendo } \\
\text { capaces de discriminar la fiabilidad de la información disponible en la web en } \\
\text { torno al tema. }\end{array}$ \\
\hline $\begin{array}{l}\text { Franco-Mariscal, Blanco-López } \\
\text { y España-Ramos (2014) }\end{array}$ & $\begin{array}{l}\text { Propuesta de una unidad didáctica de ciencias dedicada a la salud e higiene } \\
\text { bucodental que presenta diferentes tareas para desarrollar todas las } \\
\text { dimensiones de la competencia científica. Al diseñar la unidad en torno a } \\
\text { problemas de la vida diaria se pueden incorporar tareas con las que se puedan } \\
\text { desarrollar dimensiones de la competencia científica relacionadas con el } \\
\text { tratamiento de información de Internet. }\end{array}$ \\
\hline Marzo y Monferrer (2015) & $\begin{array}{l}\text { Propuesta de una experiencia educativa que pretende acercar a los estudiantes, } \\
\text { de una manera innovadora, a la materia de Física y Química y que toma de } \\
\text { referencia la metodología del aprendizaje por indagación. Se trabajan diferentes } \\
\text { herramientas de carácter didáctico, como las TIC, contribuyendo al desarrollo } \\
\text { de distintas competencias como la digital. }\end{array}$ \\
\hline Franco-Mariscal (2015) & $\begin{array}{l}\text { Planteamiento de un enfoque alternativo para el desarrollo de la competencia } \\
\text { científica en la enseñanza-aprendizaje de las Ciencias por investigación en la } \\
\text { educación secundaria. Se considera que la competencia científica tiene siete } \\
\text { dimensiones, entre ellas el manejo de la información. }\end{array}$ \\
\hline
\end{tabular}

\section{Consideraciones finales}

A modo de síntesis, podemos concluir que podemos tener distintos referentes para definir la competencia informacional, pero en todos se considera que forma parte de una más amplia, la competencia digital, que engloba no sólo elementos informativos sino la comunicación y colaboración, la creación de contenidos, la seguridad y la resolución de problemas.

Por otro lado, hemos visto que, en nuestros dos últimos programas oficiales, se ha presentado como elemento trasversal, lo que exige la identificación de cómo se puede contribuir a su adquisición desde las diferentes materias. Además, hemos podido apreciar que la última formulación oficial es más acorde con la revisión realizada. 
También hemos revisado contribuciones de la investigación sobre el grado de desarrollo competencial del alumnado de secundaria. Al respecto encontramos resultados contradictorios, pero podemos decir que, aunque los estudiantes ponen de manifiesto ciertas habilidades digitales, tienen una formación limitada e insuficiente para iniciar la búsqueda, interpretar el contenido, valorar su fiabilidad, conocer la seguridad en la red o usar éticamente la tecnología.

A pesar de la variedad de recursos existentes en las TIC, la presencia de los mismos en las aulas parece que es escasa, pero, sobre todo, se puede decir que se desaprovechan gran parte de las posibilidades que se derivan de su utilización. Parece obligado que el tratamiento escolar de la ciencia se adapte a las necesidades actuales en la formación de ciudadanos autónomos y capaces de informarse de forma crítica y objetiva en Internet para tomar decisiones y resolver problemas en contextos cotidianos que guarden relación con la ciencia y la tecnología.

Después hemos contemplado cómo diferentes autores han defendido que la competencia informacional y la competencia digital forman parte de la competencia científica; en particular, entre las necesidades derivadas de las capacidades científicas deseables en los ciudadanos, aparecen algunas relacionadas con la obtención de información, la valoración de la misma según su procedencia, de los procedimientos para manejarla y comunicarla, etc. Pero, además, para la formación propedéutica de nuevos científicos resulta fundamental, ya que las fuentes bibliográficas científicas y publicaciones de calidad y prestigio (revistas, bases de datos, webs de grupos de investigación...) se encuentran mayoritariamente en la red y son la fuente habitual de consulta para profesionales dedicados a la ciencia desde cualquiera de sus áreas (Jamali y Asadi 2010, Yeagley, Porter, Rhoten y Topham 2016).

Finalmente, hemos visto algunos ejemplos de aportaciones (propuestas, actividades, experiencias) que, al incidir en la adquisición de la competencia científica, trabajan las competencias informacional y digital. Estos trabajos aportan dos avales: por un lado, clarifica lo que recoge el currículum oficial y, por otro, aporta credibilidad o justifica la viabilidad de que es posible adquirir estas competencias en nuestras aulas de ciencias. Muchos profesores aceptan que deben incorporar a su trabajo las TIC o las competencias digitales, pero desconocen cómo hacerlo (para qué, qué se pretende cuándo, en qué momento...), lo que genera incertidumbre y dudas en su práctica habitual.

Algunas implicaciones de la adquisición de competencias científicas y digitales podrían ser:

- Conocer estrategias para la búsqueda de información en diferentes medios y desarrollar capacidades como la identificación de ideas, la constatación de semejanzas y contradicciones, la inferencia a partir de la información, etc.

- Desarrollar un pensamiento crítico al analizar la información, valorar su credibilidad, realizar posicionamientos argumentados, apreciar sus implicaciones éticas y sociales, etc.

- Desarrollar la capacidad para argumentar en torno a un tema o problema de carácter científico al emitir juicios y opiniones formadas e independientes, basadas en la información disponible en la red.

- Conocer formas de comunicación para compartir, colaborar, cooperar, interaccionar, participar, reflexionar colectivamente, etc. en comunidades escolares y sociales.

- Crear contenidos digitales (imágenes, vídeos, textos) de carácter científico o reelaborar algunos existentes para difundir lo que se sabe, se piensa, se siente..., respetando la propiedad intelectual, la privacidad, el derecho de los niños, etc.

- Hacer un uso seguro de las TIC y, en particular, de Internet. 
Por ello, las TIC por sí mismas o su uso no promueven el desarrollo de la competencia informacional-digital, ni el aprendizaje de la disciplina que se considere. Por esta razón resulta clave el conocimiento sobre distintas webs, recursos, actividades, unidades didácticas y contextos científicos donde explotar la potencialidad de las TIC para el aprendizaje de conocimientos, procedimientos o actitudes ligadas a las ciencias que permitan el desarrollo de esta competencia y de la necesaria formación de nuestros alumnos.

\section{Referencias}

Álvarez-Herrero J.F. (2010) Infoxicación y procesos de enseñanza-aprendizaje en Física y Química de $3^{\circ}$ de ESO. En Abril A.M., Quesada A. (Editores) Actas de los XXIV Encuentros de Didáctica de las Ciencias Experimentales (pp. 785-793). Baeza: Universidad de Jaén.

Ananiadou K., Claro M. (2009) 21st Century Skills and Competences for New Millennium Learners in OECD Countries, OECD Education Working Papers, 41.

Area M., Guarro A. (2012) La alfabetización informacional y digital: fundamentos pedagógicos para la enseñanza y aprendizaje competente. Revista Española de Documentación Científica, No Monográfico, 46-74.

Badia A. (2009) Enseñar a ser competente en el uso de las TIC para manejar y transformar la información en conocimiento. Aula de Innovación Educativa, 181, 13-16.

Banet E. (2010) Finalidades de la educación científica en educación secundaria: Aportaciones de la investigación educativa y opinión de los profesores. Enseñanza de las Ciencias, 28(2), 199-214.

Blanco-López A., España-Ramos E., González-García F.J., Franco-Mariscal A.J. (2015) Key Aspects of Scientific Competence for Citizenship: A Delphi Study of the Expert Community in Spain. Journal of Research in Science Teaching, 52(2), 164-198. DOI: 10.1002/tea. 21188

Blanco-López A., España-Ramos E., Franco-Mariscal A.J. (2017) Estrategias didácticas para el desarrollo del pensamiento crítico en el aula de ciencias. Ápice. Revista de Educación Cientifica, 1(1), 107-115.

Blasco A., Durban G. (2012) La competencia informacional en la enseñanza obligatoria a partir de la articulación de un modelo específico. Revista Española de Documentación Cientifica, $\mathrm{N}^{\circ}$ Monográfico, 100-135.

BOE. (2007) Real Decreto 1631/2006, de 29 de diciembre, por el que se establecen las enseñanzas mínimas correspondientes a la Educación Secundaria Obligatoria, BOE $\mathrm{n}^{\circ}$ 5, de 5 de enero de 2007.

BOE. (2015) Orden ECD/65/2015, de 21 de enero, por la que se describen las relaciones entre las competencias, los contenidos y los criterios de evaluación de la educación primaria, la educación secundaria obligatoria y bachillerato, $\mathrm{BOE} \mathrm{n}^{\circ} 25$, de 29 de enero de 2015.

Calvani A., Fini A., Ranieri M., Picci P. (2012) Are young generations in secondary school digitally competent? A study on Italian teenagers. Computers \& Education, 58, 797-807. 
Campal M. F. (2015) ¿Por qué lo llaman competencia digital, cuando quieren decir alfabetización informacional? Mi Biblioteca: La revista del mundo bibliotecario, 42, 38-43.

Cañal P. (2012) ¿Cómo evaluar la competencia científica? Investigación en la Escuela, 78, 5-17.

Colás P., Conde J., Reyes S. (2017) Competencias digitales del alumnado no universitario. Revista Latinoamericana de Tecnología Educativa, 16(1), 7-20.

Coll C. (2008) Aprender y enseñar con las TIC: expectativas, realidad y potencialidades. En Cameiro, R., Toscano, J.C., Díaz, T (coord.) Los desafíos de las TIC para el cambio educativo (pp. 113-126). Madrid: Metas Educativas de la OEI.

Colwell J., Hunt S., Reinking D. (2013) Obstacles to Developing Digital Literacy on the Internet in Middle School Science Instruction. Journal of Literacy Research, 45(3), 295324.

Cid A. (2013) Proyecto Perdiodistas intrépidos. Aprendemos a investigar y a utilizar la información. Aula de Innovación Educativa, 221, 75-81.

De Pro A. (2012) Las implicaciones sociales del conocimiento científico y tecnológico forman parte de éste y, por lo tanto, de su enseñanza. En Pedrinaci E. (coord.) Once ideas clave: El desarrollo de la competencia cientifica, (pp. 171-194). Barcelona: Graó.

Dimopoulos K., Asimakopoulos A. (2010) Science on the Web: Secondary School Student's Navigation Patterns and Preferred Page's Characteristics. Journal of Science Education and Technology, 19(3), 246-265.

European Parliament and the Council (2006) Recomendación del Parlamento Europeo y del Consejo de 18 de diciembre de 2006 sobre las competencias clave para el aprendizaje permanente. Diario Oficial de la Unión Europea, L394/10.

Ferrari A. (2013) DIGCOMP: A Framework for Developing and Understanding Digital Competence in Europe. En Y. Punie, y B.N. Brecko (Eds.), JRC scientific and policy reports. Louxembourg: Publications Office of the European Union. http://dx.doi.org/10.2788/52966

Fornás R. (2003) Criterios para evaluar la calidad y fiabilidad de los contenidos en Internet. Revista Española de Documentación Científica, 26(1), 75-80.

Franco A.J.; España E., Blanco A. (2014) Uso de Internet para analizar las relaciones entre drogas y salud bucodental. Una experiencia en $4^{\circ}$ de ESO. En Soriano E., González A.J. y Cala, V.C. (eds.). Retos actuales de educación y salud transcultural [1]. Almería: Universidad de Almería, cap. 54.

Franco-Mariscal A. J., Blanco-López A., España-Ramos E. (2014) El desarrollo de la competencia científica en una unidad didáctica sobre la salud bucodental. Diseño y análisis de tareas. Enseñanza de las Ciencias, 32(3), 649-667.

Franco-Mariscal A.J. (2015) Competencias científicas en la enseñanza y el aprendizaje por investigación. Un estudio de caso sobre la corrosión de metales en secundaria. Enseñanza de las Ciencias, 33(2), 231-252. 
FECYT (2015) VII Encuesta de percepción social de la ciencia. Dossier informativo.

FECYT (2017) VIII Encuesta de percepción social de la ciencia. Dossier informativo.

Gabby A., Avargil S., Herscovitz O., Dori Y.J. (2017) The case of middle and high school chemistry teachers implementing technology: using the concerns-based adoption model to assess changes processes. Chemistry Education Research and Practice. Advance Article. DOI: 10.1039/c6rp00193a.

Garritz A (2010) La enseñanza de la ciencia en una sociedad con incertidumbre y cambios acelerados. Enseñanza de las Ciencias, 28(3), 315-326.

Gómez M. A., Cañas A. M., Gutiérrez M. S., Martín-Díaz M. J. (2014) Ordenadores en las aulas: ¿estamos preparados los profesores? Enseñanza de las Ciencias, 32(2), 239-250.

Grimalt C., Pintó R., Ametller J. (2013) La utilización del aula digital en las clases de ciencias de secundaria. Análisis de la situación actual. Proyecto ADIGIC. Alambique: Didáctica de las Ciencias Experimentales, 75, 91-98.

Jamali H.R., Asadi S. (2010) Google and the scholar: the role of Google in scientists' information-seeking behaviour. Online Information Review, 34(2), 282-294.

Julien H., Barker S. (2009) How high-school students find and evaluate scientific information: A basis for information literacy skills development. Library \& Information Science Research, 31, 12-17.

Keck D., Kammerer Y., Starauschek E. (2015) Reading science text online: Does source information influence the identification of contradictions within texts? Computers \& Education, 82, 442-449.

López J., Blanco A., Haro G. (2011) «Ahorra energía: ¡Tú puedes!» Una unidad didáctica web para el desarrollo de la competencia científica y de la competencia digital. En Ruiz, J. y Sánchez, J. (coords.) II Congreso Internacional sobre Uso y Buenas Prácticas con TIC. Málaga: Universidad de Málaga.

Marzo A., Monferrer L. (2015) Pregúntate, indaga y a la vez trabaja algunas competencias. Revista Eureka sobre Enseñanza y Divulgación de las Ciencias, 12(1), 198-211.

Monereo C. (2005) Internet, un espacio idóneo para desarrollar las competencias básicas. En Monereo, C. (coord.) Internet y competencias básicas: Aprender a colaborar, a comunicarse, a aprender, (pp 5 - 26). Barcelona: Graó.

Monereo C., Fuentes M. (2008) Cómo buscan información en Internet los adolescentes. Investigación en la Escuela, 64, 45-58.

Monereo C. (2009) Competencia digital: para qué, quién, dónde y cómo debe enseñarse. Aula de Innovación Educativa, 181, 9-12.

Monereo C. (2010) ¡Saquen el libro de texto! Resistencia, obstáculos, y alternativas en la formación de los docentes para el cambio educativo. Revista de Educación, 352, 583-597. 
Monereo C., Badia A. (2012) La competencia informacional desde una perspectiva psicoeducativa: enseñanza basada en la resolución de problemas prototípicos y emergentes. Revista Española de Documentación Científica, No Monográfico, 75-99.

Murga-Menoyo M.A, García I. (2010) La carta de la Tierra y el blog. Dos elementos centrales de una experiencia docente innovadora en $4^{\circ}$ de ESO. Enseñanza de las Ciencias, 28(1), 85-94.

OECD. (2013) PISA 2015. Draft Science Framework.

Pedrinaci E. (2012) El ejercicio de una ciudadanía responsable exige disponer de cierta competencia científica. En Pedrinaci E. (coord.) Once ideas clave: El desarrollo de la competencia cientifica, (pp. 15-35). Barcelona: Graó.

Revuelta G., Corchero C. (2015) Acceso a la información sobre ciencia y tecnología: evolución e implicaciones. En Fundación Española para la Ciencia y la Tecnología (Ed.) Percepción Social de la Ciencia y la Tecnología 2014, (pp. 99-130). Madrid: FECYT.

Rodríguez F., Blanco A., Rueda J.A. (2011) Competencia científica y competencia digital en una unidad didáctica sobre el consumo de agua embotellada. En Ruiz J., Sánchez J. (Coords.) II Congreso Internacional sobre Uso y Buenas Prácticas con TIC. Málaga: Universidad de Málaga.

Starcevic V., Aboujaoude E. (2015) Cyberchondria, cyberbullying, cybersuicide, cybersex: «new» psychopathologies for the $21^{\text {st }}$ century? The Journal of World Psychiatry, 14(1), 97100.

Tsai M. J., Hsu C. Y., Tsai, C. C. (2012) Investigation of High School Students' Online Science Information Searching Performance: The Role of Implicit and Explicit Strategies. Journal of Science Education and Technology, 21(2), 246-254.

Valverde-Crespo D., González-Sánchez J. (2016) Búsqueda y selección de información en recursos digitales: Percepciones de alumnos de Física y Química de Educación Secundaria Obligatoria y Bachillerato sobre Wikipedia. Revista Eureka sobre Enseñanza y Divulgación de las Ciencias, 13(1), 67-83.

Valverde D., González J., De Pro A. (2017) ¿Qué sub-competencias digitales muestran unos alumnos de $4^{\circ}$ de Educación secundaria obligatoria ante una animación sobre una reacción química a nivel microscópico? Ápice. Revista de Educación Científica, 1(1), 40-57.

Vuorikari R., Punie Y., Carretero Gomez S., Van den Brande G. (2016) DigComp 2.0: The Digital Competence Framework for Citizens. Update Phase 1: The Conceptual Reference Model. Luxembourg Publication Office of the European Union. EUR 27948 EN. Doi:10.2791/11517

Walraven A., Brand-Gruwel S., Boshuizen H. (2009) How students evaluate information and sources when searching in the World Wide Web for information. Computers \& Education, 52, 234-246.

Yeagley A. A., Porter S. E., Rothen M. C., Topham B. J. (2016) The Stepping Stone Approach to Teaching Chemical Information Skills. Journal of Chemical Education, 93(3), 423-428. 